\title{
Higher retinal vessel oxygen saturation: investigating its relationship with macular oedema in retinitis pigmentosa patients
}

\author{
Rossiana I. Bojinova ${ }^{1,2}$ - Daniel F. Schorderet $\mathbb{1}^{3,4,5} \cdot$ Christophe Valmaggia $^{6}$ - Cengiz Türksever ${ }^{1,7}$. \\ Andreas Schoetzau ${ }^{1} \cdot$ Margarita G. Todorova $^{1}$
}

Received: 12 February 2017 / Revised: 20 January 2018 / Accepted: 28 January 2018 / Published online: 6 March 2018

(c) The Royal College of Ophthalmologists 2018

\begin{abstract}
Purpose Primary objective - to investigate the effect of retinal vessel oxygen saturation $\left(\mathrm{SO}_{2}\right)$ on macular oedema (ME) in retinitis pigmentosa (RP) patients. Secondary objective-to link the presence of ME to metabolic (oxygen saturation of retinal vessels, $\mathrm{SO}_{2}$ ), functional (multifocal electroretinography, mfERG) and structural (Spectral Domain Optical Coherent Tomography, SD-OCT) alterations in RP.

Design Prospective, cross-sectional, non-interventional study.

Subjects Patients with typical RP $(N=37)$ and controls $(N=19)$, who underwent retinal vessel Oximetry (RO), SD-OCT and mfERG, were included.

Methods A computer-based program of the retinal vessel analyser unit (IMEDOS Systems UG, Jena, Germany) was used to measure $\mathrm{SO}_{2}$. We evaluated the mean $\mathrm{SO}_{2}$, in all major retinal arterioles (oxygen saturation in retinal arterioles, $\mathrm{A}-\mathrm{SO}_{2}, \%$ ) and venules (oxygen saturation in retinal venules, $\mathrm{V}-\mathrm{SO}_{2}, \%$ ). MfERG responses were averaged in zones (zone $1\left(0-3^{\circ}\right)$, zone $2\left(3-8^{\circ}\right)$ and zone $3\left(8-15^{\circ}\right)$ ) and compared to corresponding areas of the OCT. The effect of $\mathrm{ME}$ on $\mathrm{SO}_{2}$ was evaluated dividing the RP in two subgroups: with clinical appearance of ME (ME-RP) and without it (no-ME-RP).

Main outcome measures Parallel recording and juxtaposition of metabolic $\left(\mathrm{SO}_{2}\right)$ to structural (OCT) and functional$(\mathrm{mfERG})$ measures. Mean $( \pm \mathrm{SD}) \mathrm{A}-\mathrm{SO}_{2}$ and $\mathrm{V}-\mathrm{SO}_{2}$ were higher in no-ME-RP $(96.77 \%( \pm 6.31)$ and $59.93 \%( \pm 7.76))$ and even higher in the ME-RP $(99.82 \%( \pm 6.21)$ and $65.63 \%( \pm 7.63))$, compared to controls $(93.15 \%( \pm 3.76)$ and $53.77 \%$ $( \pm 3.70), p \leq 0.006)$.

Results The subgroup ME-RP differed significantly from the subgroup no-ME-RP by increased A-SO $\mathrm{S}_{2}$ and $\mathrm{V}-\mathrm{SO}_{2}, p \leq$ 0.026. The presence of ME confirmed a different relationship between the altered $\mathrm{SO}_{2}$ and the vessel diameters, against the functional and structural parameters.

Conclusion Based on our results, the presence of macular oedema indicates a tendency toward greater alteration of the metabolic function in RP patients.
\end{abstract}

Electronic supplementary material The online version of this article (https://doi.org/10.1038/s41433-018-0043-1) contains supplementary material, which is available to authorized users.

Margarita G. Todorova

margarita.todorova@usb.ch

1 Department of Ophthalmology, University of Basel, Mittlere Strasse 91, Basel CH-4031, Switzerland

2 University of Montreal, 495 Prince Arthur West, Montreal H2X1T4, Canada

3 IRO-Institute for Research in Ophthalmology, Sion, Switzerland

\section{Introduction}

Retinitis pigmentosa (RP) constitutes a heterogeneous group of inherited retinal diseases, marked by progressive photoreceptor cell degeneration. With disease progression,

4 Department of Ophthalmology, University of Lausanne, Lausanne, Switzerland

5 School of Life Sciences, Federal Institute of Technology, Lausanne, Switzerland

6 Department of Ophthalmology, Cantonal Hospital, St. Gallen, Switzerland

7 VISTA Klinik, Binningen, Baselland, Switzerland 
Table 1 Demographic data of RP patients and controls

\begin{tabular}{|c|c|c|c|}
\hline \multirow[t]{2}{*}{ Demographic characteristics } & \multicolumn{2}{|c|}{ Retinitis pigmentosa patients } & \multirow[t]{2}{*}{ Controls } \\
\hline & no-ME-RP subgroup & ME-RP subgroup & \\
\hline Number of subjects (eyes) & $25(44)$ & $18(30)$ & $19(38)$ \\
\hline Mean age (standard error) (years) & $40.89(1.51)$ & $40.90(1.82)$ & $38.00(1.62)$ \\
\hline Sex: প/ठ̋ & $11 / 14$ & $9 / 9$ & $13 / 6$ \\
\hline
\end{tabular}

Six patients were part of both RP subgroups (three right and three left eyes per RP subgroup)

only a central island of functioning photoreceptors remains, at which stage the patient is left with tunnel vision. The degenerative process involves initially the rods, with later involvement of the cones. As the cones become affected, the remaining central visual function deteriorates further, leading to blindness in many patients [1-5].

Oxygen is known to be the most extensively supplied metabolite in the retina [6,7]. Recent studies of RP in animal models have demonstrated a marked reduction of oxygen utilisation with acceleration of the rod photoreceptor degeneration. However, the choroidal blood flow is not auto-regulated. As the oxygen delivery of the outer retina remains unchanged, the intraretinal oxygen level increases steadily. With disease progression, a measured increase of superoxide radicals in both the mitochondria and the cytoplasm of the cones follows [8]. Furthermore, the excess of superoxide radicals seems to generate other reactive oxygen species, resulting in even greater oxidative damage in the cones [9].

Since using antioxidants in RP models supposedly decreases the oxidative damage and prevents the cone photoreceptors' death, it is inferred that the oxidative damage is a major contributor to cone photoreceptor apoptosis [10].

Retinal vessel oximetry (RO) is a novel research method, measuring retinal vessels' oxygen saturation in vivo. In patients affected by inherited retinal diseases, retinal vessel oximetry (RO) showed an alteration of the oxygen metabolism with severe increase in retinal vessel oxygen saturation [11-16]. Furthermore, the oxygen saturation values correlated well not only with the structural alterations [12, 16] (detected by optical coherence tomography (OCT)) but also with the functional alterations [17] (detected by full-field electroretinography (ERG), electrooculography (EOG) and multifocal electroretinography (mfERG)). Since the highest amount of oxygen is used by the retinal photoreceptors, a reduction of retinal oxygen demand with secondary increase of the retinal vessel saturation values following cellular apoptosis has been proposed to explain the observed vascular oxygen alterations in RP patients. Correspondingly, peripapillary retinal vessel diameters were reduced proportionally to the functional [18] and structural alterations [16]. Thus, degeneration of the photoreceptors with secondary neurovascular remodelling seems to be a causative factor of the increased retinal vessel saturation in RP [12, 17, 18].

The relationship between the residual cone-mediated responses (as per mfERG recordings) and the structural alterations (as per OCT data) on one hand and the visual function in patients with RP, on the other hand, has been examined before by a number of studies [19-23]. For instance, the integrity of the photoreceptor inner/outer segment junction, labelled as IS/OS line on OCT imaging, has been strongly associated with better visual function and higher retinal sensitivity [24-26]. The correlations between the best corrected visual acuity and the structure-function parameters were noticeably more pronounced within the RP subgroup without clinical appearance of macular oedema (no-ME-RP, retinitis pigmentosa subgroup without macular oedema) [27]. Surprisingly, the RP subgroup with clinical appearance of macular oedema (ME-RP, retinitis pigmentosa subgroup with macular oedema), even with better preservation of the central vision, showed more advanced stage of photoreceptor degeneration with greater disruption of the IS/OS line, and greater attenuation of the central mfERG responses. [27] However, to what extent the presence of macular oedema corresponds to the retinal vessel oxygen saturation $\left(\mathrm{SO}_{2}\right)$ and retinal vessel attenuation in RP patients, has not been studied yet. Applying the metabolic-structure-function approach, we aim to investigate the relationship between the presence of macular oedema $(\mathrm{ME})$ and the metabolic $\left(\mathrm{SO}_{2}\right)$, functional (mfERG) and structural (OCT) alterations in patients with RP.

\section{Subjects and methods}

\section{Subjects}

Demographic data of the RP patients and controls are given in Table 1. We included 37 consecutive patients with RP (74 eyes) and recruited 19 age-matched controls (38 eyes). The age range was $20-60$ years (mean: $40.89 \pm 10.85$ years) and 29-51 years (mean: $38.00 \pm 7.82$ years), $(p=0.148)$, of RP patients and controls, respectively. All subjects were 


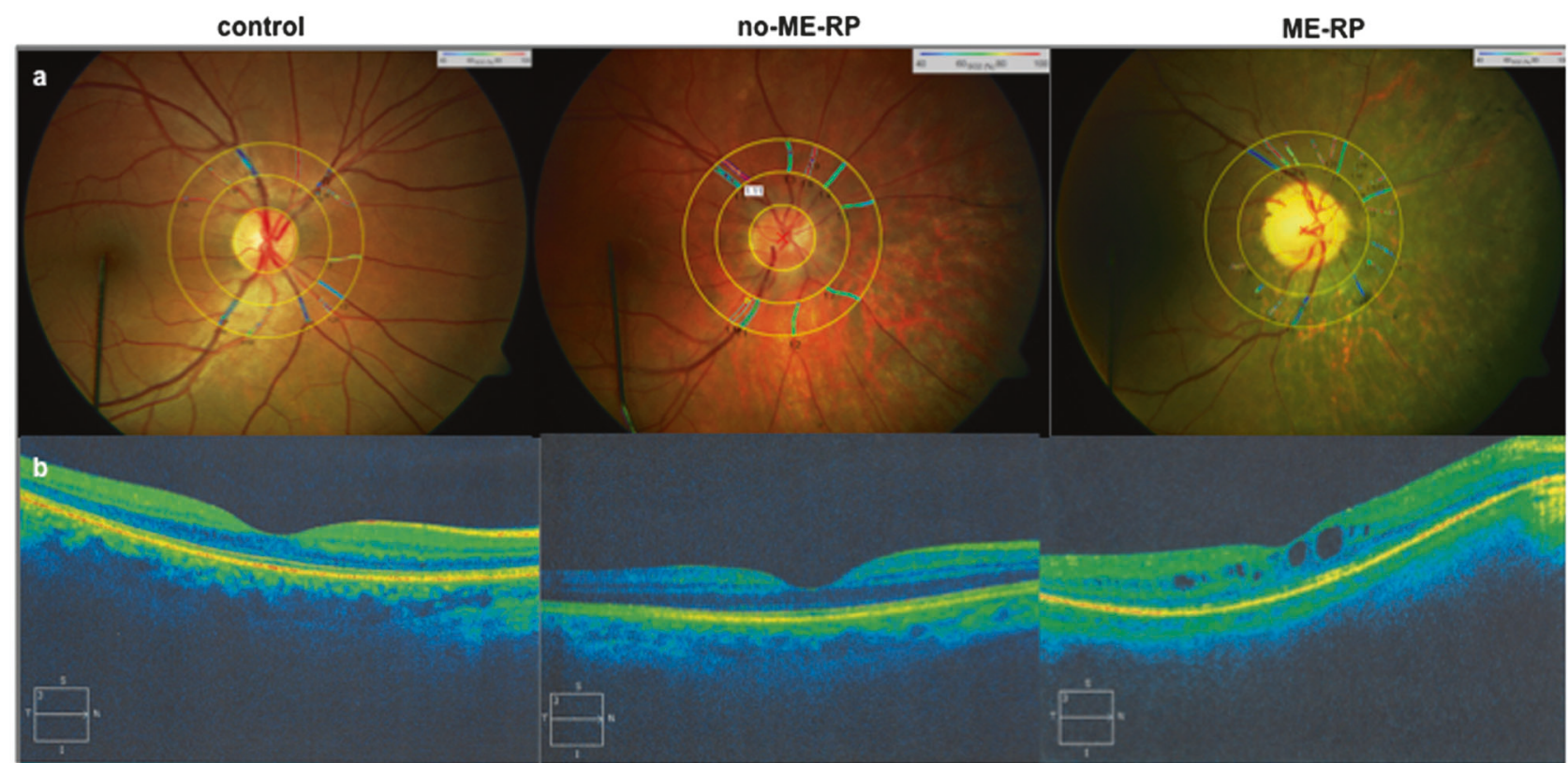

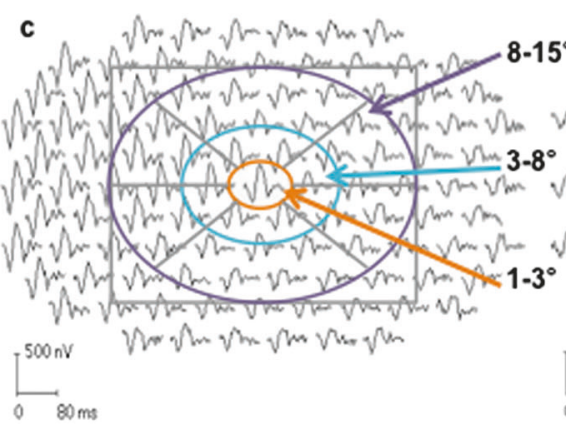

Fig. 1 Retinal vessel oximetry images (RO) from the right eye of a control subject (right), a representative no-ME-RP patient (middle) and a ME-RP patient (ME-RP; left) (a). Optical coherence tomography

examined at the diagnostic unit of the Department of Ophthalmology at the University of Basel. The study was approved by the Ethics Commission of Central and Northern Switzerland, EKNZ Basel Switzerland (trial number EKNZ BASEC 2016-01054). The tenets of the Declaration of Helsinki were followed throughout the study.

All controls and RP patients underwent standard ophthalmologic examination, including best corrected visual acuity (Snellen charts), Goldmann applanation tonometry measurements, biomicroscopy and fundoscopy. The clinical phenotype of RP patients was characterised following clinical and electrophysiological assessment.

The inclusion criteria for RP patients were: Caucasian origin, characteristic funduscopic findings of RP, reduced or scotopic negative full-field ERG. The inclusion criteria for controls were: Caucasian origin and best corrected visual acuity at distance $\geq 0.8$.

Exclusion criteria for RP patients and controls, were: history of surgical treatment (cataract surgery inclusively) on the examined eye, clinical signs of macular pathology images (SD-OCTs) from the right eye of the same subjects (b). MfERG tracings from the right eye of a control subject and RP patients (c)

other than RP, systemic diseases (such as diabetes mellitus, hypertension, and/or other metabolic and neurodegenerative diseases, potentially affecting the OCT imaging, mfERG recording or RO measurements), history of antidepressant use, alcohol and/or drug consumption.

\section{Retinal vessel oximetry imaging}

Both pupils of each subject were dilated to $7.0-8.0 \mathrm{~mm}$ using Tropicamide $0.5 \%$ and Phenylephrine $1 \%$ eye drops. After a minimum of $20 \mathrm{~min}$, having reached a pupil dilation of at least $7.0 \mathrm{~mm}$, measured on slit lamp biomicroscopy (BI $900^{\circ}$, Haag-Streit, Koeniz, Switzerland), four test-retest fundus images were obtained, as described previously [11, $13,18,28]$. Briefly, optic disc fundus images, with $50^{\circ}$ field span, were taken for each eye using the Retinal Vessel Analyser (RVA: Imedos UG, Jena, Germany) which was connected to the fundus camera FF450 (Carl Zeiss Meditec, Jena, Germany). Images of both eyes were obtained, starting with the right eye. 


\section{Image analyses}

Optic disc-centred image protocol was applied for analysis, where two concentric rings are created in the peripapillary area: one with a radius of 1.0 optic disc diameters, and second with a radius of 1.5 optic disc diameters. The region between both circles defined the area of interest. All main arterioles and venules within the measurement area were manually selected for analysis of the mean arteriolar and venular oxygen saturation $\left(\mathrm{A}-\mathrm{SO}_{2}\right.$ and $\left.\mathrm{V}-\mathrm{SO}_{2}\right)$, and their corresponding diameters (D-A and $\mathrm{D}-\mathrm{V}, \mu \mathrm{m})$. The arteriovenular difference in oxygen saturation $\left(\mathrm{A}-\mathrm{V} \mathrm{SO} \mathrm{SO}_{2}\right)$, known to be proportional to the oxygen saturation of the retina, was calculated as well. Representative images of a control and two RP patients are given in Fig. 1a.

\section{Optical coherence tomography (OCT)}

For the evaluation of the retinal structure, we performed a spectral domain optical coherence tomography (SD-OCT) using Cirrus OCT (Carl Zeiss Meditec. Dublin, CA, USA). The OCT images were taken by implementing macular thickness protocol (Macular Cube $512 \times 128$ ) and high definition image-protocol (HD 5 Line Raster). Both, vertical and horizontal scan OCT images from each RP patient were screened for clinical appearance of macular oedema, by looking for the presence of visible intraretinal spaces situated at the central macula $(1.5 \mathrm{~mm}$ in diameter; Fig. 1b). In addition, we measured manually the length of the intact IS/OS line for every eye on HD 5 Line Raster OCT images, using a measurement tool from the Cirrus OCT.

\section{Multifocal electroretinogram (mfERG)}

We used VERIS ${ }^{\text {TM }} 6.0$ (Electro-Diagnostic Imaging, San Mateo, CA, USA) for the mfERG recording. During the recording session 103 hexagons (Fig. 1) flickered between black and white according to m-sequence of $2^{\wedge} 15$ (frame rate: $75 \mathrm{~Hz})$. The central retina $\left(50^{\circ}\right.$ around the centre) was stimulated. During the light phase the maximal luminance of the hexagons $\left(\mathrm{L}_{\max }\right)$ was $200 \mathrm{~cd} / \mathrm{m}^{2}$ and during the dark phase $\left(\mathrm{L}_{\mathrm{min}}\right)$ was $<1 \mathrm{~cd} / \mathrm{m}^{2}$. The retinal signals were bandpass-filtered at $10-200 \mathrm{~Hz}$. The responses were recorded monocularly with a single-use microfiber DTL electrode (DTL Plus Electrode, Diagnosis LLC). The negative electrode was placed on the temporal orbital rim and the ground electrode - on the forehead. If a refractive error was present, corrections were done using the FMS III fundus stimulator (EDI, San Mateo, CA).

We measured the N1 response amplitude of the mfERG from the baseline to the trough of the first negative wave (N1), while we estimated the N1P1 response amplitude from the trough of the first negative wave (N1) to the peak of the first positive wave (P1). Corresponding to the macular thickness map protocol, provided by the software of the Cirrus OCT, mfERG responses were averaged in three concentric rings, i.e., zones: around the foveola, or zone 1 (within the central $3^{\circ}$; orange), fovea, or zone 2 (between $3^{\circ}$ and $8^{\circ}$; turquoise) and paracentral area, or zone 3 (between $8^{\circ}$ and $15^{\circ}$; violet) (Fig. 1c, left-hand side).

\section{Genetic analyses}

Genetic testing was performed in 18 out of 35 RP patients. Blood samples were taken from the 18 participants and genomic DNA was extracted from blood leukocytes, according to a standard procedure. Molecular analysis was performed using an in-house developed in-solution capture assay of the most frequent RP genes, followed by highthroughput sequencing. All mutations were validated by Sanger sequencing using primers [29], obtained with the Primer3 software (http://primer3.ut.ee) [30, 31]. In two additional RP patients genetic analyses were previously completed in another institution.

\section{Statistical analysis}

IBM SPSS Statistics version 21 (International Business Machines Corp., Armonk, NY, USA) was applied for statistical analysis.

In order to explore the association of functional and structural parameters (mfERG and OCT) with the metabolic parameters (RO), linear mixed-effects models were performed.

Mixed effects models were suitable regression tools to analyse repeated measure data (here applied for the left and right eye).

RO parameters were dependent variables, while age, gender, average amplitude of the mfERG (nV/ $\left./ \mathrm{deg}^{2}\right)$, macular thickness $(\mu \mathrm{m})$, IS/OS length $(\mu \mathrm{m})$ and/or study groups were independent variables. Subject was treated as a random factor. As a result of the inclusion of age and gender in the models, all outcomes were adjusted for these parameters.

Results are presented as mean differences between study groups or regression slopes with corresponding 95\% confidence intervals $(\mathrm{CI})$ and $p$-values.

In addition, potential interactions between study groups and functional or structural parameters were included in the regression model. A statistically significant interaction of functional or structural parameters with a study group would mean, that the association of these parameters with RO variables will be different between study groups (regression slopes are different). $p$-Values $>0.05$ were considered as statistically significant. 
Table 2 Descriptive statistics for the parameters evaluated in RP groups and in the control group

\begin{tabular}{|c|c|c|c|c|}
\hline \multirow[t]{4}{*}{ Parameters } & \multirow{4}{*}{$\begin{array}{l}\text { Controls } \\
\text { Adjusted means, based on } \\
\text { mixed-effects models } \\
\text { (standard error) }\end{array}$} & \multicolumn{2}{|c|}{ Retinitis pigmentosa patients } & \multirow{4}{*}{$\begin{array}{l}\mathrm{p} \text {-values } \\
\text { (ANOVA, based on } \\
\text { mixed-effects models) } \\
p \text {-values }\end{array}$} \\
\hline & & \multicolumn{2}{|c|}{ Mean difference in subgroups ( $p$-values) } & \\
\hline & & $\begin{array}{l}\text { Controls vs. no- } \\
\text { ME-RP subgroup }\end{array}$ & $\begin{array}{l}\text { Controls vs. } \\
\text { ME-RP } \\
\text { subgroup }\end{array}$ & \\
\hline & & no-ME-RP v & P subgroup & \\
\hline \multicolumn{5}{|l|}{$B C V A$} \\
\hline \multirow[t]{3}{*}{ Snellen charts } & $1.00(0.04)$ & $0.436(0.06)$ & & $<0.001 *$ \\
\hline & & & $0.482(0.06)$ & $<0.001 *$ \\
\hline & & & & 0.449 \\
\hline \multicolumn{5}{|c|}{ Retinal vessel oxygen saturation (\%) } \\
\hline \multirow[t]{3}{*}{$\mathrm{A}-\mathrm{SO}_{2}$} & $93.15(0.96)$ & $-3.62(1.29)$ & & 0.006* \\
\hline & & & $-6.67(1.41)$ & $<0.001 *$ \\
\hline & & -3 & & $0.026^{*}$ \\
\hline \multirow[t]{3}{*}{$\mathrm{V}-\mathrm{SO}_{2}$} & $53.77(1.15)$ & $-6.15(1.54)$ & & $<0.001 *$ \\
\hline & & & $-11.86(1.69)$ & $<0.001 *$ \\
\hline & & -5 & & $0.001 *$ \\
\hline \multirow[t]{3}{*}{$\mathrm{A}-\mathrm{V} \mathrm{SO} \mathrm{SO}_{2}$} & $39.38(1.26)$ & $2.53(1.69)$ & & 0.137 \\
\hline & & & $5.19(1.85)$ & $0.006^{*}$ \\
\hline & & & & 0.136 \\
\hline \multicolumn{5}{|c|}{ Retinal vessel diameter $(\mu m)$} \\
\hline \multirow[t]{3}{*}{ D-A } & $91.85(2.22)$ & $5.13(2.86)$ & & 0.088 \\
\hline & & & $9.10(3.27)$ & $0.006 *$ \\
\hline & & & & 0.207 \\
\hline \multirow[t]{3}{*}{$\mathrm{D}-\mathrm{V}$} & $118.69(2.68)$ & $14.57(3.94)$ & & $<0.001 *$ \\
\hline & & & $15.23(3.60)$ & $0.001 *$ \\
\hline & & & & 0.862 \\
\hline \multicolumn{5}{|c|}{ OCT thickness $(\mu \mathrm{m})$} \\
\hline \multirow[t]{3}{*}{ Zone 1} & $261.87(10.14)$ & $33.55(13.98)$ & & 0.018* \\
\hline & & & $-50.72(15.61)$ & $0.002 *$ \\
\hline & & -84 & & $<0.001 *$ \\
\hline \multirow[t]{3}{*}{ Zone 2} & $317.97(7.34)$ & $39.43(10.13)$ & & $<0.001 *$ \\
\hline & & & $-7.74(11.31)$ & 0.495 \\
\hline & & -47 & & $<0.001 *$ \\
\hline \multirow[t]{3}{*}{ Zone 3} & $269.96(5.64)$ & $25.82(7.78)$ & & $<0.001 *$ \\
\hline & & & $5.75(8.69)$ & 0.510 \\
\hline & & -20 & & $0.020 *$ \\
\hline \multirow[t]{3}{*}{ IS/OS line } & $5981.32(236.29)$ & 3777.29 (329.96) & & $<0.001 *$ \\
\hline & & & $\begin{array}{l}3004.82 \\
(379.79)\end{array}$ & $<0.001 *$ \\
\hline & & -772 & .09) & $0.043 *$ \\
\hline \multicolumn{5}{|l|}{ mfERG } \\
\hline \multicolumn{5}{|c|}{ N1 amplitudes (nV/deg $\left.{ }^{2}\right)$} \\
\hline \multirow[t]{3}{*}{ Zone 1} & $-61.16(2.52)$ & $-42.15(3.57)$ & & $<0.001 *$ \\
\hline & & & $-36.72(3.95)$ & $<0.001 *$ \\
\hline & & & & 0.105 \\
\hline Zone 2 & $-34.66(1.31)$ & $-24.72(1.85)$ & & $<0.001 *$ \\
\hline
\end{tabular}


Table 2 (continued)

\begin{tabular}{|c|c|c|c|c|}
\hline \multirow[t]{4}{*}{ Parameters } & \multirow{4}{*}{$\begin{array}{l}\text { Controls } \\
\text { Adjusted means, based on } \\
\text { mixed-effects models } \\
\text { (standard error) }\end{array}$} & \multicolumn{2}{|c|}{ Retinitis pigmentosa patients } & \multirow{4}{*}{$\begin{array}{l}\mathrm{p} \text {-values } \\
\text { (ANOVA, based on } \\
\text { mixed-effects models) } \\
p \text {-values }\end{array}$} \\
\hline & & \multicolumn{2}{|c|}{ Mean difference in subgroups ( $p$-values) } & \\
\hline & & $\begin{array}{l}\text { Controls vs. no- } \\
\text { ME-RP subgroup }\end{array}$ & $\begin{array}{l}\text { Controls vs. } \\
\text { ME-RP } \\
\text { subgroup }\end{array}$ & \\
\hline & & \multicolumn{2}{|c|}{ no-ME-RP vs. ME-RP subgroup } & \\
\hline & & & $-20.20(2.05)$ & $<0.001 *$ \\
\hline & & & & $0.029 *$ \\
\hline \multirow[t]{3}{*}{ Zone 3} & $-17.20(0.66)$ & $-13.19(0.92)$ & & $<0.001 *$ \\
\hline & & & $-10.08(1.02)$ & $<0.001 *$ \\
\hline & & & & $0.003 *$ \\
\hline \multicolumn{5}{|c|}{ N1P1 amliptudes (nV/deg $\left.{ }^{2}\right)$} \\
\hline \multirow[t]{3}{*}{ Zone 1} & $139.34(5.16)$ & $99.58(7.2)$ & & $<0.001 *$ \\
\hline & & & $83.98(8.03)$ & $<0.001 *$ \\
\hline & & -1 & & 0.054 \\
\hline \multirow[t]{3}{*}{ Zone 2} & $75.45(2.72)$ & $56.99(3.82)$ & & $<0.001 *$ \\
\hline & & & $46.96(4.23)$ & $<0.001 *$ \\
\hline & & -1 & & $0.019 *$ \\
\hline \multirow[t]{3}{*}{ Zone 3} & $35.88(1.37)$ & $28.66(1.92)$ & & $<0.001 *$ \\
\hline & & & $24.87(2.13)$ & $<0.001 *$ \\
\hline & & & & 0.076 \\
\hline
\end{tabular}

Statistically significant p-values $(<0.05)$ are given in bold

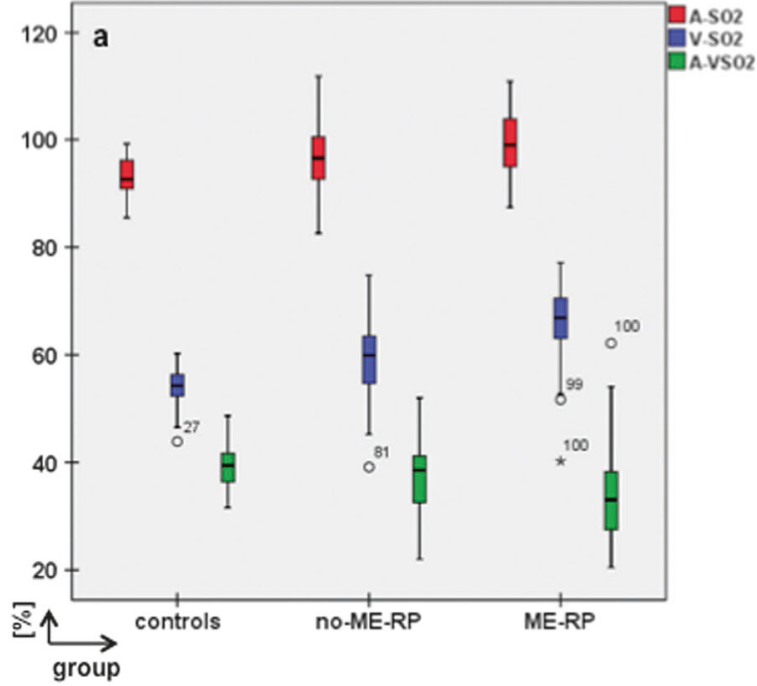

Fig. 2 Box plots of the retinal vessels oxygen saturation for arterioles $\left(\mathrm{A}-\mathrm{SO}_{2}\right)$, venules $\left(\mathrm{V}-\mathrm{SO}_{2}\right)$ and their difference $\left(\mathrm{A}-\mathrm{V} \mathrm{SO}_{2}\right)(\mathbf{a})$, as well as of the retinal vessel diameters for the respective arterioles (D-A) and venules $(\mathrm{D}-\mathrm{V})(\mathbf{b})$. The box length is the interquartile range, the line in

\section{Power calculation}

To the best of our knowledge, there has been no previous investigation of the retinal oximetry values in RP patients

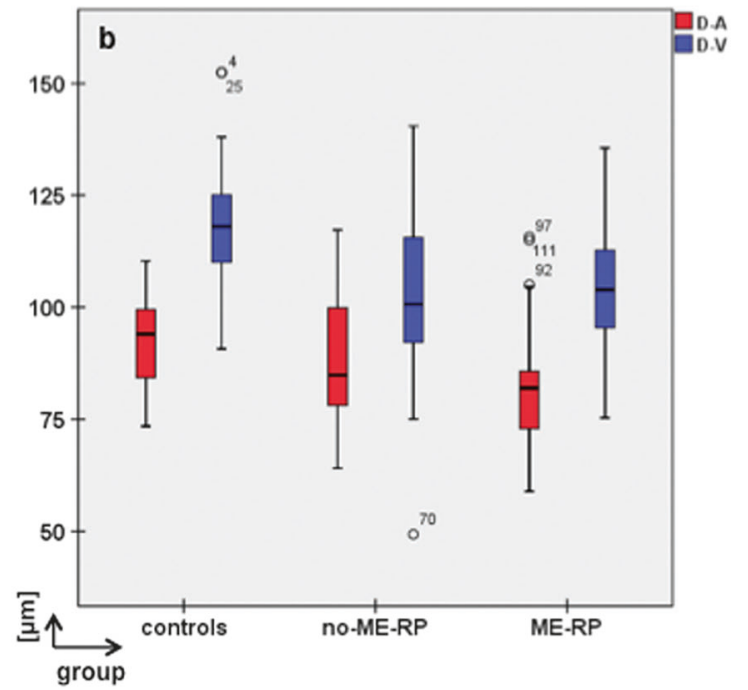

bold depicts the median. In each graph, the control group is plotted to the left, the no-ME-RP subgroup is plotted in the middle, and the ME$\mathrm{RP}$ subgroup is plotted to the right. The respective $\mathrm{p}$-values are given in Table 2 (ANOVA, based on linear mixed-effects model)

with or without presence of macular oedema. Therefore, the present study was designed as an exploratory study, no statistical hypothesis was formulated and no formal sample size calculation was done. 
Fig. 3 Linear mixed-effects model is applied to correlate the oxygen saturation measurements (a $\mathrm{A}-\mathrm{SO}_{2} ; \mathbf{b} \mathrm{V}-\mathrm{SO}_{2}$ and $\mathbf{c} \mathrm{A}-\mathrm{V}$ $\mathrm{SO}_{2}$ ) to the $\mathrm{N} 1$ amplitudes of the mfERG (left-hand side) to the N1P1 amplitudes of the mfERG (middle), and to the OCT thickness measurements (righthand side), as well as the retinal vessel diameter measurements (d D-A; e D-V) to the N1 amplitudes of the mfERG (lefthand side), to the N1P1 amplitudes of the mfERG (middle), and to the OCT thickness measurements (righthand side). Scatter-plots with their corresponding regression lines are presented as individual graphs: on the top for controls, in the middle for no-ME-RP patients, and on the bottom for ME-RP patients. The oxygen saturation data $(\%)$ and the peripapillary retinal vessel diameter data $(\mu \mathrm{m})$ are plotted on the $Y$-axis and the N1 mfERG data $\left(\mathrm{nV} / \mathrm{deg}^{2}\right)$, the N1P1 data $\left(\mathrm{nV} / \mathrm{deg}^{2}\right)$, and respectively the OCT thickness data $(\mu \mathrm{m})$ are plotted on the $X$-axis (orange: zone 1, turquoise: zone 2 , and purple: zone 3)
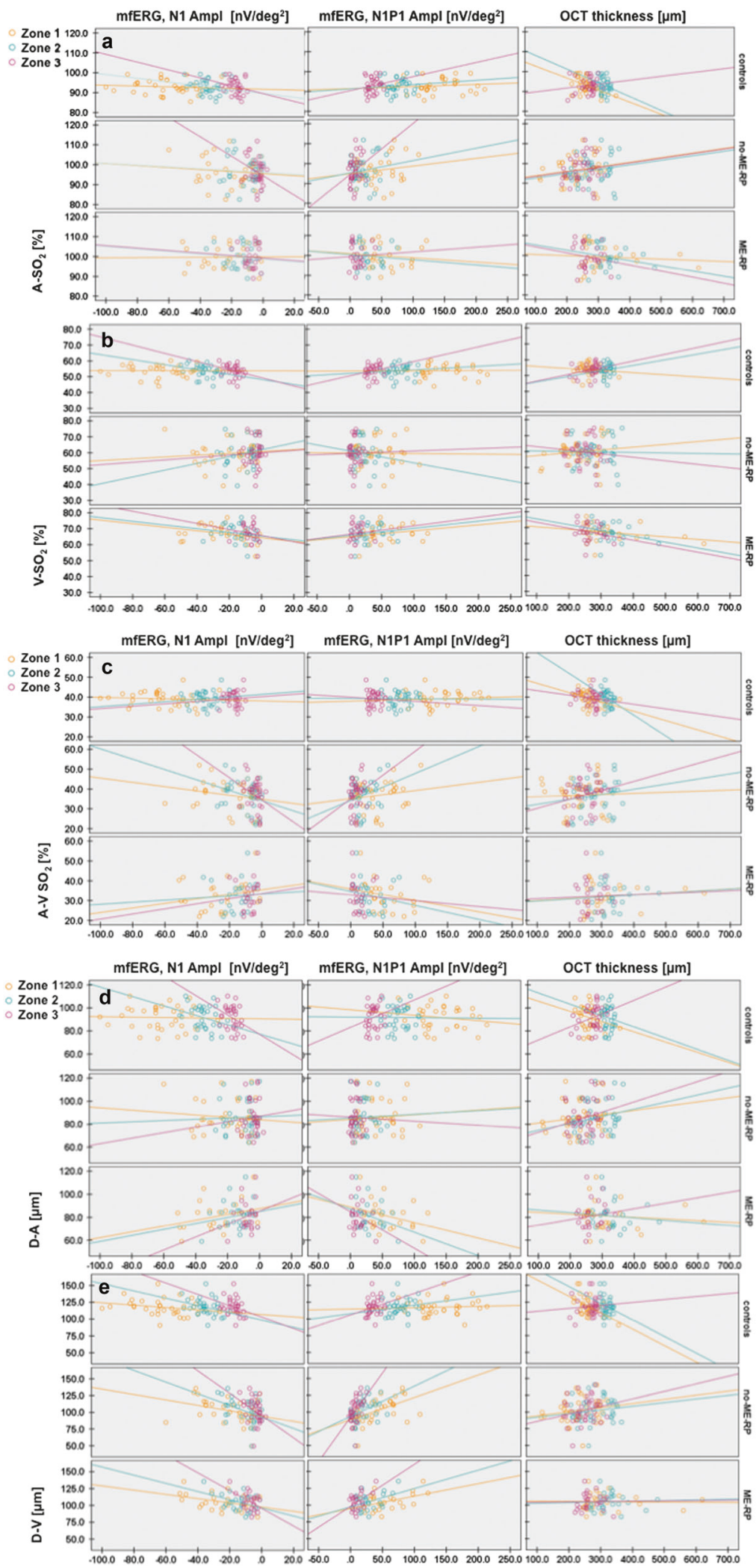


\section{Results}

A total of 37 patients (20 female and 17 male; 74 eyes) with clinical and electrophysiological evidence of retinitis pigmentosa were enroled in the study. In total 19 age-matched controls (13 female and 6 male; 38 eyes) without RP were recruited from among our clinic patients. The RP group had two subgroups: with (ME-RP) and without appearance of macular oedema (no-ME-RP), defined by the presence of intraretinal spaces situated within the foveal centre. In the no-ME-RP subgroup 25 patients participated with 44 eyes (22 right and 22 left) without macular oedema, and in the ME-RP subgroup 18 patients participated with 30 eyes (15 right and 15 left) with macular oedema. Six patients were part of both subgroups, each having one eye with ME (3 right and 3 left eyes). Both RP subgroups showed different mutations, affecting different genes, including simplex and syndromic cases with an autosomal-dominant, autosomal-recessive and X-linked mode of inheritance (supplement Table).

Retinal vessel oximetry results: increased $\mathrm{A}-\mathrm{SO}_{2}, \mathrm{~V}-\mathrm{SO}_{2}$ and decreased $\mathrm{A}-\mathrm{V} \mathrm{SO} \mathrm{S}_{2}$ values in ME-RP patients

In general, RP patients had higher mean $\mathrm{A}-\mathrm{SO}_{2}$ and $\mathrm{V}-\mathrm{SO}_{2}$ values when compared to controls $(p \leq 0.006$, ANOVA based on mixed-effect models, Table 2, Fig. 2). Evaluated in subgroups, ME-RP patients differed from controls, but also from no-ME-RP patients, when the mean $\mathrm{A}-\mathrm{SO}_{2}$ and $\mathrm{V}-\mathrm{SO}_{2}$ values were compared $(p \leq 0.026$, Table 2, adjusted means, based on mixed-effect models). The corresponding mean A-V SO $\mathrm{S}_{2}$ decreased slightly by $2.53 \%$ in the ME-RP $(p=0.137)$ but significantly by $5.19 \%$ in the no-ME-RP subgroup ( $p=0.006$, Table 2$)$, when compared to controls.

\section{Retinal vessel diameter results: narrower D-A and D- $V$ in RP patients}

The mean peripapillary retinal vessel diameters were narrower in the RP group than in controls ( $p \leq 0.006$; Table 2). Noticeably, the ME-RP subgroup showed narrower mean peripapillary arterial diameters, when compared to the noME-RP subgroup (Table 2, Fig. 2).

\section{Functional and structural findings in RP patients}

Within the RP group, eyes clinically classified as no-ME$\mathrm{RP}$ showed a greater impairment of the central retinal function than the ME-RP patients, compared to controls. The latter was measured by means of $\mathrm{N} 1$ and N1P1 amplitudes of the mfERG (Table 2). Depending on the presence of macular oedema, the retinal thickness evaluated in all zones was reduced in the no-ME-RP $(p \leq 0.018)$, while it was increased in zone 1 of the ME-RP $(p=0.002)$, when compared to controls (Table 2). The OCT imaging confirmed loss of photoreceptors and distortions of the retinal microstructure (impaired IS/OS line integrity) within the entire RP group. Even if the length of the IS/OS line was more reduced in the no-ME-RP subgroup $(p=0.023$, Table 2), within the ME-RP subgroup the IS/OS line was more disrupted toward the foveola, than in the no-ME RP subgroup.

\section{Relationship between structural/functional parameters and the RO-parameters within study groups}

Aiming to predict the effect (relationship) of structural/ functional parameters on RO parameters, linear mixedeffects models were created, including interactions between study groups.

In linear mixed effect models, a significant interaction indicates a different relationship of functional/structural parameters with the RO-variables between study groups. As a whole, in our study, interactions between functional/ structural and RO parameters, and study groups were present (Fig. 3a-e).

For instance, as $\mathrm{A}-\mathrm{SO}_{2}$ increased, N1 and N1P1 mfERG amplitudes (evaluated in zones) within the no-ME-RP subgroup decreased, whereas in the ME-RP subgroup the corresponding regression slops were not significant (Fig. 3a). Further, as the $\mathrm{A}-\mathrm{SO}_{2}$ increased, the retinal thickness within the no-ME-RP subgroup increased, whereas in the ME-RP subgroup and in controls decreased (Fig. 3a). Also, as the $\mathrm{V}-\mathrm{SO}_{2}$ values increased, the N1mfERG and N1P1 mfERG amplitudes, within the MERP subgroup, decreased and the retinal thickness decreased, whereas in the no-ME subgroup the corresponding regression slops were not significant (Fig. 3b).

Interactions between venular retinal vessel diameter and functional alterations (mfERG data) were also significant between study groups. For instance, as the diameter of arterioles and venules got narrower, the retinal thickness in the no-ME-RP subgroup decreased, whereas in the ME-RP subgroup the corresponding regression slops showed no significant trend (Fig. 3d). In both RP subgroups, as the diameter of venules got narrower, the N1 and N1P1 mfERG amplitudes became significantly lower ( $p \leq 0.042$; Fig. 3e).

\section{Discussion}

In agreement with previous reports [11-17], the present study confirms, that retinal vessel oxygen saturation is altered in patients with retinitis pigmentosa and shows that the metabolic function in patients with clinical appearance of macular oedema is even more affected. 
Altered retinal oxygen metabolism has been confirmed through in vivo research on animal models of outer retinal degeneration, where changes in the oxygen environment are discussed as significant players in the progression of the degenerative process [32-35]. Reflecting a decreased metabolic demand in the degenerating retina, we also observed an increased $\mathrm{A}-\mathrm{SO}_{2}$, and even more, an increased $\mathrm{V}-\mathrm{SO}_{2}$ with a corresponding decrease of the A-V $\mathrm{SO}_{2}$ values within the RP group. The respective values were significantly higher in the ME-RP subgroup.

Some authors have hypothesised that the process of retinal remodelling, occurring as the retina degenerates [3639], is responsible for the thickening of retinal layers in eyes with RP [40, 41]. Vigorous retinal remodelling is observed once photoreceptor loss occurs: novel synapse formation, microneuroma formation, Müller cell hypertrophy, and amacrine cells, as well as bipolar cell inversion [38]. As these phenomena are thought to contribute to the clinical appearance of macular oedema, we divided our RP patients into two subgroups, differentiated one from the other by the clinical appearance of macular oedema. A novel finding in the present study is that those RP patients with clinical appearance of macular oedema had even more affected metabolic function. ME-RP patients were clearly differentiated from controls, but also from no-ME-RP patients, when venular and arterial oxygen saturation were taken into account. Both the mean $\mathrm{A}-\mathrm{SO}_{2}$ - and the mean $\mathrm{V}-\mathrm{SO}_{2}$ values were significantly higher in the ME-RP subgroup.

There are several possible explanations for our findings. An RP remodelling process has been linked to the stage of cone survival [38, 41], where metabolic dysfunction and oxidative stress are found to correlate with cone dysfunction and death [42] However, direct comparison with the animal models is restricted, as the human topographic distribution of photoreceptors differs from that of animals. Outside the fovea, animal models show the same topography as the human retina, with almost $90 \%$ of photoreceptors being rods. Inside the fovea, the cone distribution differs between humans and animal models. Compared to human photoreceptors' topography, showing higher cone density in the fovea [43], rodents do not have a macula per se. Even if cones are thought to be the main players in the pathogenesis of the outer retinal degeneration, the presence of macular oedema has not been studied in relation to oxygen utilisation in rodent models of outer retinal degeneration, so far. Nevertheless, taking the results of our study, it is plausible, that the simultaneous presentation of macular oedema and increased retinal vessel oxygen saturation reflects the compromised resistance of cones to oxidative damage following rods' apoptosis in humans. An argument in favour of that is the fact, that both RPsubgroups showed different metabolic, structural and functional alterations.
Interestingly, in our study ME-RP patients had less preserved N1 and N1P1 responses of the mfERG, thus being presumed to have greater alteration of the cone-mediated function. Noticeably, ME-RP patients, even with more preserved central vision (by means of best-corrected visual acuity), have shown more advanced stage of photoreceptor degeneration [24]. All of the above leaves an open question of whether the two RP subgroups present a different stage of the progressive degenerative process. The ME-RP subgroup seems to represent more decimating RP stage, marked by more aggressive remodelling of the neural retina and thus, more significantly altered metabolic function. Nonetheless, in our patients, genetic analyses revealed a variety of mutations affecting different genes, where in some cases, a unilateral, instead of bilateral macular oedema was present (suppl. Table).

Neural, glial, as well as vascular remodelling of the remaining retina accompanies retinal degeneration [44]. In agreement with this finding we confirmed narrower peripapillary vessels within the RP group, when compared to controls. Again, the ME-RP subgroup showed even narrower peripapillary vessels and significantly higher retinal vessels oxygen saturation values, than the no-ME-RP subgroup. Furthermore, depending on the presence of macular oedema within the RP group, our results indicated a different relationship between the altered oxygen saturation and vessel diameter parameters against the functional and structural parameters.

The study by Kim et al. confirmed the severity of macular oedema in RP patients to correlate with the IS/OS line disruptions. Clearly, eyes with disrupted IS/OS junctions exhibited greater total retinal thickness and thinner photoreceptor layer, compared to eyes with intact IS/ OS junctions [25]. In addition the photoreceptor IS/OS length and the outer retinal thickness in the fovea are found to correlate to retinal functions such as: visual field dimension, visual acuity, and ERG amplitudes [24, 27, 45, 46]. Our study also found reduced IS/OS length within the RP group. The shorter and more disrupted the IS/OS junctions were, the higher the oxygen saturation values were measured.

When evaluated in subgroups, depending on the presence of macular oedema, our results indicated a different relationship between the altered oxygen saturation and vessel diameter parameters against the functional and structural parameters. Here, within the ME-RP subgroup, the interactions were more pronounced when evaluated against venular saturation, with the $\mathrm{V}-\mathrm{SO}_{2}$ values being higher as the retinal thickness increased and the mfERG amplitudes decreased (Fig. 3a).

Furthermore, we found for both RP subgroups a significant association between the venular diameter (D-V) and the functional alterations (mfERG data). The interactions between the retinal vessel diameter data and the retinal function were statistically significant for all examined 
zones: the mfERG amplitudes got lower, as the $\mathrm{D}-\mathrm{V}$ diameters became narrower.

The role of the oxidative stress is discussed as a trigger for cones and inner retina cell loss, once the rod photoreceptors die $[8,9]$. However, not only a local retinal oxidative stress, but also a systemic oxidative stress [47, 44] should be evaluated in the pathogenetic chain of events in RP through further studies.

\section{Summary}

In conclusion, our study showed that RP patients with macular oedema have a greater alteration of the metabolic function. Increased retinal vessel oxygen saturation, narrower retinal vessels, and more disrupted IS/OS lines are linked to the clinical appearance of macular oedema. Altogether, we suggest that, as the photoreceptors die during the progressive degenerative process, the normal metabolic homoeostasis in the RP retina suffers significant alteration. This leads to higher levels of oxygen free radicals in the intercellular space, causing persistent macular thickening, clinically identified as macular oedema. Whether a more advanced cone decimating RP stage is marked by more aggressive remodelling of the neural retina and, thus, by even more altered metabolic function, remains to be investigated. Further studies are also needed, to evaluate to what extend the altered retinal and choroidal perfusion in RP patients may contribute to the metabolic dysfunction and to the presence of macular oedema.

\section{Summary}

\section{What was known before}

- Retinal vessel oxygen saturation is altered in retinitis pigmentosa patients.

\section{What this study adds}

- Metabolic function in RP patients, with clinical appearance of macular oedema, is even more affected.

- Depending on the presence of macular oedema within the RP group, our results indicated a different relationship between altered oxygen saturation- and vessel diameter parameters against functional and structural parameters.

\section{Data access and responsibility}

Margarita G. Todorova has full access to all the data in the study and holds complete responsibility for the data integrity and the accuracy of the analysis.
Acknowledgements Margarita G. Todorova was partially supported by unrestricted grant from OPOS (Stiftung Ostschweizerische Pleoptik- and Orthoptik-Schule) and by unrestricted grant from LHW (Liechtenstein Stiftung). We are extremely thankful to Nathaniel Jordan Lee; his technical support was invaluable for accomplishing our work.

\section{Compliance with ethical standards}

Conflict of interest The authors declare that they have no conflict of interest.

\section{References}

1. Ammann F, Klein D, Franceschetti A. Genetic and epidemiological investigations on pigmentary degeneration of the retina and allied disorders in Switzerland. J Neurol Sci. 1965;2:183-96.

2. Puech B, Kostrubiec B, Hache JC, François P. Epidemiology and prevalence of hereditary retinal dystrophies in the Northern France. J Fr Ophtalmol. 1991;14:153-64.

3. Hamel C. Retinitis pigmentosa. Orphanet J Rare Dis. 2006;1:40.

4. Hamel C. Cone rod dystrophies. Orphanet J Rare Dis. 2007;1:7.

5. Michaelides M, Hunt DM, Moore AT. The genetics of inherited macular dystrophies. J Med Genet. 2003;40:641-50.

6. Anderson B Jr, Saltzman HA. Retinal Oxygen utilisation measured by hyperbaric blackout. Arch Ophthalmol. 1964;72:792-5.

7. Wangsa-Wirawan ND, Linsenmeier RA. Retinal oxygen: fundamental and clinical aspects. Arch Ophthalmol. 2003;121:547-57.

8. Usui S, Oveson BC, Lee SY, Jo YJ, Yoshida T, Miki A, Miki K, Iwase T, Lu L, Campochiaro PA. NADPH oxidase plays a central role in cone cell death in retinitis pigmentosa. J Neurochem. 2009;110:1028-37.

9. Shen J, Yang X, Dong A, Petters RM, Peng YW, Wong F, Campochiaro PA. Oxidative damage is a potential cause of cone cell death in retinitis pigmentosa. J Cell Physiol. 2005;203:457-64.

10. Komeima K, Rogers BS, Lu L, Campochiaro PA. Antioxidants reduce cone cell death in a model of retinitis pigmentosa. Proc Natl Acad Sci USA. 2006;103:11300-5.

11. Todorova MG, Türksever C, Schorderet DF, Valmaggia C. Retinal vessel Oxygen Saturation in patients suffering from inherited diseases of the retina. Klin Monbl Augenheilkd. 2014;231:447-52.

12. Türksever C, Valmaggia C, Orgül S, Schorderet DF, Flammer J, Todorova MG. Retinal vessel oxygen saturation and its correlation with structural changes in retinitis pigmentosa. Acta Ophthalmol. 2014;92:454-60.

13. Eysteinsson T, Hardarson SH, Bragason D, Stefánsson E. Retinal vessel oxygen saturation and vessel diameter in retinitis pigmentosa. Acta Ophthalmol. 2014;92:449-53.

14. Ueda-Consolvo T, Fuchizawa $\mathrm{C}$, Otsuka M, Nakagawa $\mathrm{T}$, Hayashi A. Analysis of retinal vessels in eyes with retinitis pigmentosa by retinal oximeter. Acta Ophthalmol. 2015;93: e446-450.

15. Zong Y, Lin L, Yi C, Huang X, Fu Y, Dong Y, Qian X, Li Y, Gao $\mathrm{Q}$. Retinal vessel oxygen saturation and vessel diameter in retinitis pigmentosa at various ages. Graefes Arch Clin Exp Ophthalmol. 2016;254:243-52.

16. Bojinova RI, Türksever C, Schötzau A, Valmaggia C, Schorderet DF, Todorova MG. Reduced metabolic function and structural alterations in inherited retinal dystrophies: investigating the effect of peripapillary vessel oxygen saturation and vascular diameter on the retinal nerve fibre layer thickness. Acta Ophthalmol. 2017;95:252-61. 
17. Todorova MG, Türksever C, Schötzau A, Schorderet DF, Valmaggia $\mathrm{C}$. Metabolic and functional changes in retinitis pigmentosa: comparing retinal vessel oximetry to full-field electroretinography, electrooculogram and multifocal electroretinography. Acta Ophthalmol. 2016;94:e231-41.

18. Lopez Torres LT, Türksever C, Schötzau A, Orgül S, Todorova MG. Peripapillary retinal vessel diameter correlates with mfERG alterations in retinitis pigmentosa. Acta Ophthalmol. 2015;93: e527-33.

19. Sandberg MA, Weigel-DiFranco C, Rosner B, Berson EL. The relationship between visual field size and electroretinogram amplitude in retinitis pigmentosa. Invest Ophthalmol Vis Sci. 1996;37:1693-8.

20. Gerth C, Wright T, Héon E, Westall CA. Assessment of central retinal function in patients with advanced retinitis pigmentosa. Invest Ophthalmol Vis Sci. 2007;48:1312-8.

21. Ma Y, Kawasaki R, Dobson LP, Ruddle JB, Kearns LS, Wong TY, Mackey DA. Quantitative analysis of retinal vessel attenuation in eyes with retinitis pigmentosa. Invest Ophthalmol Vis Sci. 2012;53:4306-14.

22. Nagy D, Schönfisch B, Zrenner E, Jägle H. Long-term follow-up of retinitis pigmentosa patients with multifocal electroretinography. Invest Ophthalmol Vis Sci. 2008;49:4664-71.

23. Wen Y, Klein M, Hood DC, Birch DG. Relationships among multifocal electroretinogram amplitude, visual field sensitivity, and SD-OCT receptor layer thicknesses in patients with retinitis pigmentosa. Invest Ophthalmol Vis Sci. 2012;53:833-40.

24. Aizawa S, Mitamura Y, Baba T, Hagiwara A, Ogata K, Yamamoto S. Correlation between visual function and photoreceptor inner/outer segment junction in patients with retinitis pigmentosa. Eye. 2009;23:304-8.

25. Kim YJ, Joe SG, Lee DH, Lee JY, Kim JG, Yoon YH. Correlations between spectral-domain OCT measurements and visual acuity in cystoid macular edema associated with retinitis pigmentosa. Invest Ophthalmol Vis Sci. 2013;54:1303-9.

26. Mitamura Y, Mitamura-Aizawa S, Katome T, Naito T, Hagiwara A, Kumagai K, Yamamoto S. Photoreceptor impairment and restoration on optical coherence tomographic image. J Ophthalmol. 2013;2013:518170

27. Konieczka K, Bojinova RI, Valmaggia C, Schorderet DF, Todorova MG, Medscape. Preserved functional and structural integrity of the papillomacular area correlates with better visual acuity in retinitis pigmentosa. Eye. 2016;30:1310-23.

28. Türksever C, Orgül S, Todorova MG. Reproducibility of retinal oximetry measurements in healthy and diseased retinas. Acta Ophthalmol. 2015;93:e439-45.

29. Schorderet DF, Iouranova A, Favez T, Tiab L, Escher P. IROme, a new high-throughput molecular tool for the diagnosis of inherited retinal dystrophies. Biomed Res Int. 2013;2013:198089.

30. Koressaar T, Remm M. Enhancements and modifications of primer design program Primer3. Bioinformatics. 2007; 23:1289-91.
31. Untergasser A, Cutcutache I, Koressaar T, Ye J, Faircloth BC, Remm M, Rozen SG. Primer3-new capabilities and interfaces. Nucleic Acids Res. 2012;40:e115.

32. Yu DY, Cringle SJ. Oxygen distribution and consumption within the retina in vascularised and avascular retinas and in animal models of retinal disease. Prog Retin Eye Res. 2001;20:175-208.

33. Yu DY, Cringle SJ. Retinal degeneration and local oxygen metabolism. Exp Eye Res. 2005;80:745-51.

34. Padnick-Silver L, Kang Derwent JJ, Giuliano E, Narfström K, Linsenmeier RA. Retinal oxygenation and oxygen metabolism in Abyssinian cats with a hereditary retinal degeneration. Invest Ophthalmol Vis Sci. 2006;47:3639-3689.

35. Yu DY, Cringle SJ, Su EN, Yu PK. Intraretinal oxygen levels before and after photoreceptor loss in the RCS rat. Invest Ophthalmol Vis Sci. 2000;41:3999-4006.

36. Milam AH, Li ZY, Fariss RN. Histopathology of the human retina in retinitis pigmentosa. Prog Retin Eye Res. 1998;17:175-205.

37. Panfoli I, Calzia D, Bianchini P, Ravera S, Diaspro A, Candiano G, Bachi A, Monticone M, Aluigi MG, Barabino S, Calabria G, Rolando M, Tacchetti C, Morelli A, Pepe IM. Evidence for aerobic metabolism in retinal rod outer segment disks. Int J Biochem Cell Biol. 2009;41:2555-65.

38. Marc RE, Jones BW. Retinal remodeling in inherited photoreceptor degenerations. Mol Neurobiol. 2003;28:139-47.

39. Jones BW, Marc RE. Retinal remodeling during retinal degeneration. Exp Eye Res. 2005;81:123-37.

40. Li ZY, Kljavin IJ, Milam AH. Rod photoreceptor neurite sprouting in retinitis pigmentosa. J Neurosci. 1995;15:5429-38.

41. Jones BW, Watt CB, Frederick JM, Baehr W, Chen CK, Levine EM, Milam AH, Lavail MM, Marc RE. Retinal remodeling triggered by photoreceptor degenerations. J Comp Neurol. 2003;464:1-16.

42. Punzo C, Xiong W, Cepko CL. Loss of daylight vision in retinal degeneration: are oxidative stress and metabolic dysregulation to blame? J Biol Chem. 2012;287:1642-8.

43. Kostic C, Arsenijevic Y. Animal modelling for inherited central vision loss. J Pathol. 2016;238:300-10.

44. Jones BW, Pfeiffer RL, Ferrell WD, Watt CB, Marmor M, Marc RE. Retinal remodeling in human retinitis pigmentosa. Exp Eye Res. 2016;150:149-65.

45. Fischer MD, Fleischhauer JC, Gillies MC, Sutter FK, Helbig H, Barthelmes D. A new method to monitor visual field defects caused by photoreceptor degeneration by quantitative optical coherence tomography. Invest Ophthalmol Vis Sci. 2008;49:3617-21.

46. Sugita T, Kondo M, Piao CH, Ito Y, Terasaki H. Correlation between macular volume and focal macular electroretinogram in patients with retinitis pigmentosa. Invest Ophthalmol Vis Sci. 2008;49:3551-8.

47. Campochiaro PA, Strauss RW, Lu L, Hafiz G, Wolfson Y, Shah SM, Sophie R, Mir TA, Scholl HP. Is there excess oxidative stress and damage in eyes of patients with retinitis pigmentosa? Antioxid Redox Signal. 2015;23:643-8. 